\title{
17AEP-GA, an HSP90 antagonist, is a potent inhibitor of glioblastoma cell proliferation, survival, migration and invasion
}

\author{
KATARZYNA MIEKUS, JACEK KIJOWSKI, MAŁGORZATA SEKUŁA and MARCIN MAJKA \\ Department of Transplantation, Polish-American Institute of Pediatrics, \\ Jagiellonian University School of Medicine, Cracow, Poland
}

Received March 29, 2012; Accepted July 2, 2012

DOI: $10.3892 /$ or.2012.1996

\begin{abstract}
Glioblastoma multiforme (GBM) is the most frequent and the most malignant human brain tumor. The expression of receptor tyrosine kinase MET and its ligand hepatocyte growth factor (HGF) is strongly increased in GBM, where they promote tumor proliferation, cell survival, migration, invasion and angiogenesis. We used geldanamycins (GAs) (inhibitors of HSP90) in order to block glioblastoma growth and HGF-dependent cell migration and invasion. The effect of GAs on three GBM cell lines was tested and we found their antiproliferative effect on tumor cells. The maximum level of inhibition reached $70 \%$. After treatment with GAs, cells also became apoptotic as determined by Annexin V-positive staining and activation of the caspase-3 pathway. We examined the expression and activity of the MET receptor on GBM cell lines and we observed phosphorylation of AKT and MAPK after HGF stimulation by western blot analysis. Since GBM cells express high level of MET receptor and were shown to respond to HGF by increased motility we tested if GAs could negatively affect GBM cell movement. In our study, we found that GAs inhibited the chemotaxis of glioblastoma cells toward the hepatocyte growth factor gradient. The GAs also blocked migration of tumor cells through a Matrigel layer in invasion assays. The strongest inhibitory effect was observed for GA and its analog, 17AEP-GA. Based on our results, GAs, particularly 17AEP-GA, could be considered as a new potential agent to treat glioblastoma multiforme.
\end{abstract}

\section{Introduction}

Glioblastoma multiforme (GBM) is a rare tumor but one of the most frequent and the most malignant among brain tumors.

Correspondence to: Dr Katarzyna Miekus or Professor Marcin Majka, Department of Transplantation, Polish-American Institute of Pediatrics, Jagiellonian University School of Medicine, Wielicka Street 265, Cracow 30-663, Poland

E-mail:kmiekus@cm-uj.krakow.pl

E-mail:mmajka@cm-uj.krakow.pl

Key words: glioblastoma, geldanamycins, MET receptor, proliferation, migration
Despite recent therapeutic advances the prognosis still remains very poor, with most patients $(>70 \%)$ dying within 1 year after diagnosis. Five-year overall survival is $2 \%$ (1). Currently available treatment options, such as surgery, radiotherapy and chemotherapy, can only minimally prolong life expectancy.

The GBM risk factors include e.g. chemicals, pesticides and therapeutic ionizing radiation (2). In one study, children irradiated during leukemia treatment had increased risk of brain tumors including GBM $(3,4)$. Passive smoking also increases risk of GBM (5).

GBM tumors consist of poorly differentiated oncogenic astrocytes, and are often heterogenic and highly invasive (6). The loss of PTEN, EGF receptor and changes in the expression of PDGF receptors, p16 and p53 are found in GBM (7). GBM tumors have been shown to express MET receptor and its ligand hepatocyte growth factor (HGF) (8).

MET has been reported as one of the receptors responsible for proliferation, inhibition of apoptosis and dissemination of cancer cells. It is encoded by MET proto-oncogene with tyrosine kinase activity (9). MET activation by its ligand HGF leads to pleiotropic biological effects on various target cells, including the induction of cell proliferation, morphogenetic transformation, cell motility and invasiveness under both normal and pathological conditions (9-11). In transformed tissues, the activation of MET by HGF triggers tumor growth, invasion and metastasis $(9,12)$.

Both HGF and MET are overexpressed in human GBM, and it was shown that levels of expression are upregulated during transition from low grade to malignant GBM and positively correlate with GBM malignancy grade and vascularity (8). In vitro, $\mathrm{HGF}$ stimulates GBM and endothelial cell migration and proliferation as well as endothelial tube formation. The overexpression of HGF and/or MET promotes GBM growth and angiogenesis in vivo (8).

The heat shock protein 90 (HSP90) plays a key role in the process of protein folding, stabilization and degradation. It is a molecular chaperone that regulates the maturation and intracellular trafficking of several signaling proteins and receptors, including Her2/Neu, mutant EGFR, steroid receptors, AKT, RAF, SRC, mutant p53, Bcr-Abl and MET receptor $(13,14)$. In cancer cells, HSP90 is present entirely in multichaperone complexes with high ATPase activity, being involved in the processing of oncoproteins critical to cancer progression (15). 
Geldanamycin, a naturally occurring benzoquinone ansamycin antibiotic produced by yeast, and its analogues belong to a new class of anticancer agents that inhibit the molecular chaperone HSP90. Ansamycin antibiotics bind to the ATP pocket of HSP90, altering the conformation of the multichaperone complex (16). Consequently, HSP90dependent protein folding and maturation are arrested and proteins are targeted for degradation. In cancer cells, HSP90 inhibitors (geldanamycin, radicicol, herbimycin A, and 17-allylamino-17-demethoxygeldanamycin (17-AAG)), promote the degradation of HSP90-dependent oncoproteins, eventually leading to cell cycle arrest and cell death (17). Interestingly, geldanamycin derivatives accumulate and bind preferentially to HSP90 in tumor cells compared with normal cells, promoting selective cancer cell death. These properties make this class of agents suitable for clinical development as anticancer therapeutic agents (18).

In this study we evaluated the use of GAs as a potential treatment option for GBM patients. We investigated the influence of GAs on GBM growth and HGF-dependent motility of tumor cells.

\section{Materials and methods}

Cell lines and cell culture. Experiments were performed on three human glioblastoma cell lines (LN18, LN229, T98G). Cells were kindly provided by Dr K. Reiss from Temple University, (Philadelphia, PA, USA). The cells were cultured in DMEM (LN18, LN229) and in MEM (T98G) (PAA), supplemented with $100 \mathrm{IU} / \mathrm{ml}$ penicillin and $100 \mu \mathrm{g} / \mathrm{ml}$ streptomycin (Polfa Tarchomin, Poland) in the presence of $10 \%$ FBS (PAA) at $37^{\circ} \mathrm{C}, 5 \%$ of $\mathrm{CO}_{2}$ and $95 \%$ humidity.

HSP90 inhibitors. Geldanamycin, 17-AAG (17-allylamino-17-demethoxygeldanamycin), a GA analogue, both insoluble in $\mathrm{H}_{2} \mathrm{O}$ and soluble in DMSO. 17DMAG [17-(dimethylaminoethylamino)-17-demethoxygeldanamycin], a water-soluble 17-AAG analogue; 17AEP-GA [17-(2-(pyrrolidin-1-yl)ethyl)aminno-17-demethoxygeldanamycin], a water-soluble GA analogue containing an alkylamino group instead of the methoxy moiety at C17; 17DMAP-GA [17-(dimethylaminopropylamino)-17-demethoxygeldanamycin], a water-soluble GA analogue synthesized based on binding affinity to HSP90. GA, 17AAG, 17DMAG, 17AEP-GA and 17DMAP-GA were purchased from InvivoGen, Inc. (USA).

MTT assay. The influence of GAs (InvivoGen, Inc.) on viability/proliferation was checked by CellTiter $96^{\circledR}$ AQueous One Solution assay (Promega). Cells were seeded in growth medium (containing $10 \%$ FBS) on 96 -well plates at $1 \times 10^{3} \%$ well (LN18 and LN229) and at 2x10\%3/well (T98G) density in the presence of different concentration of GAs $(1,10,100$, $1,000 \mathrm{nM}$ ). After 24, 48, 72 and 96 h, $20 \mu \mathrm{l}$ of CellTiter $96^{\circledR}$ Aqueous One Solution reagent was added to each well and plates were incubated for $3 \mathrm{~h}$. Subsequently, plates were read at $490 \mathrm{~nm}$ using the ELx800 Universal Microplate Reader (BioTek) and analyzed with KC4 v3.0 with PowerReports software.

Apoptosis. The influence of GAs on glioblastoma cells was studied by staining with Annexin $\mathrm{V}$ according to the manu- facturer's instructions. Briefly, after drug treatments for 24, 48 and $72 \mathrm{~h}$, cells were stained with propidium iodide (PI) and Annexin V-FITC in $100 \mu \mathrm{l}$ of staining solution at room temperature for $15 \mathrm{~min}$ in the dark. Samples were diluted with binding buffer and were analyzed within $1 \mathrm{~h}$. In some experiments cells were tested for the activity of caspase-3 (BD Pharmingen). For this staining, cells were permeabilized for $20 \mathrm{~min}$, washed and subsequently stained with anti-caspase3-PE mAb for 30 min on ice (both Abs from BD Pharmingen). Cytofluorometric analysis was performed by FACS Canto (Becton-Dickinson) and analyzed with FASC Diva software (Becton-Dickinson).

RNA extraction and reverse transcription. Total RNA was extracted using RNeasy mini kit (Qiagen), followed by DNAse treatment (Promega). The reverse polymerase transcription was performed using M-MLV reverse transcriptase (Invitrogen) according to the manufacturer's protocol.

Quantitative real-time PCR analysis. The detection of MET mRNA level was performed by quantitative real-time PCR analysis on ABI PRISM ${ }^{\circledR} 7300$ Sequence Detection System (Applied Biosystems, Inc.) using a commercially available TaqMan PCR Master mix and primers for human MET gene (Hs01565589_m1; Applied Biosystems, Inc.). The mRNA expression level for all samples was normalized to the housekeeping gene GAPDH (Hs99999905_m1; Applied Biosystems, Inc.)

Western blot analysis. Western blot analyses were carried out on extracts prepared from GBM cells. The cells were lysed on ice with MPER lysing buffer (Pierce) containing protease and phosphatase inhibitors (Sigma). Extracted proteins were separated on a $12 \%$ sodium dodecyl sulfate-polyacrylamide gel electrophoresis (SDS-PAGE gel) and transferred to a PVDF membrane (Bio-Rad). The activation of AKT and MAPK was detected using mouse and rabbit phospho-specific antibodies (AKT, MAPK; Cell Signaling Technology, Inc.) followed by HRP-conjugated goat anti-mouse IgG or goat antirabbit IgG as secondary antibodies (Santa Cruz Biotechnology, Inc.). The membranes were developed with an ECL reagent and exposed to HyperFilm (Amersham Life Sciences). An equal loading in the lanes was evaluated by probing with an anti-GAPDH antibody (Santa Cruz Biotechnology, Inc.).

Chemotaxis studies. The direct migration of GBM cells towards HGF gradient was studied using modified Boyden's chamber with $8-\mu \mathrm{m}$ pore polycarbonate membrane inserts. The cells were seeded into the upper chamber of a Transwell inserts (Costar Transwell). The lower chamber was filled with medium containing $0.5 \% \mathrm{BSA}$ and $\mathrm{HGF}(20 \mathrm{ng} / \mathrm{ml})$. After $24 \mathrm{~h}$, inserts were removed from transwells and cells were fixed with methanol. Cells that transmigrated to the lower side of the membrane were stained with Wright solution (Merck) and counted under high power field (HPF) with an inverted microscope.

Invasion assay. The ability of GBM cells to invade was examined by BD BioCoat ${ }^{\mathrm{TM}}$ Matrigel invasion inserts (Becton-Dickinson) where cells migrated through Matrigel 
layer towards HGF gradient. After $24 \mathrm{~h}$, cells that invaded the Matrigel were counted on the undersides of filters after fixation and staining with Wright solution. As a control of invasion the same number of control inserts (no GFR Matrigel coating) were used.

Flow cytometry. The presence of MET receptor on the surface of target cells were demonstrated by staining with rabbit antiMET-FITC antibody (R\&D Systems) and analyzed by FACS. Briefly, $1 \times 10^{5}$ cells were suspended in $100 \mu \mathrm{l}$ of staining buffer (PBS, 2\% FBS) and antibody was added. Next, the cells were incubated in the dark for $30 \mathrm{~min}$ at $4^{\circ} \mathrm{C}$. The stained cells were washed, collected using FACSCanto cytometer (BectonDickinson, USA) and analyzed with FACS Diva software (Becton-Dickinson, USA).

Statistical analysis. All results are mean \pm SD. Statistical analysis was performed using the two-tailed Student's t-test. P-values $<0.05$ were considered as significant, unless otherwise indicated.

\section{Results}

Geldanamycin and its analogs inhibit proliferation of glioblastoma cells. Several in vitro studies indicate that GAs inhibit the proliferation of a wide range of cancer cells $(18,19)$. The effects of GAs on the growth rate of GBM cell lines (LN18, LN229, T98G) were determined using the CellTiter $96^{\circledR}$ Aqueous One Solution assay at $96 \mathrm{~h}$. GAs were used in 1 and $10 \mathrm{nM}$ concentrations. As shown in Fig. 1, GAs inhibited growth of all investigated cells already at the concentration of $1 \mathrm{nM}$. However, the level of inhibition differs between cell lines and geldanamycin analogs. We observed the strongest inhibition for LN18 and LN229 cell lines where all investigated analogs were able to inhibit cell growth (Fig. 1A and B). Moreover, we noted similar level of inhibition after 96-h incubation for higher concentration of GAs, 100 and 1,000 nM for these two cell lines (data not shown). However, T98G seemed to be less sensitive to investigated analogs at the concentration of 1 and $10 \mathrm{nM}$ (Fig. 1C). Growth of T98G cell line was inhibited by $50 \%$ in comparison to untreated control after incubation with 100 and 1,000 $\mathrm{nM}$ of GAs (data not shown). The best results for all investigated cell lines were obtained using 17-AEP-GA.

Induction of glioblastoma cell apoptosis by HSP90 inhibitors. We became interested whether the inhibition of cell growth is due to the induction of apoptosis. Therefore, in order to answer this question we used Annexin V and activated caspase-3 staining to detect apoptotic cells after GAs treatment. After incubation with 10 and $100 \mathrm{nM}$ of 17-AAG and 17-AEP-GA cells became Annexin V positive (Fig. 2A). These data were confirmed with caspase-3 staining. We observed a high number of cells positive for activated caspase-3 after 17-AAG and 17-AEP-GA treatment at the concentration of $100 \mathrm{nM}$ (Fig. 2B).

Glioblastoma cell lines express active MET receptor. The expression of MET receptor mRNA was estimated by realtime PCR and we have observed the expression of MET gene for all tested cell lines (Fig. 3A). LN229 cell line, which is the most malignant one, had the highest expression of mRNA for

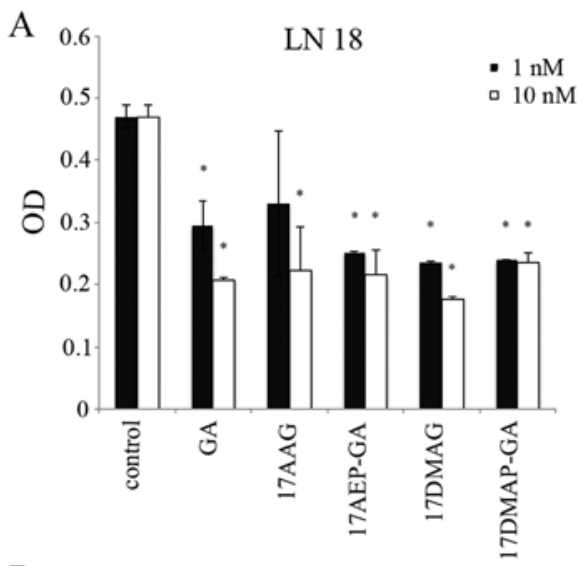

B
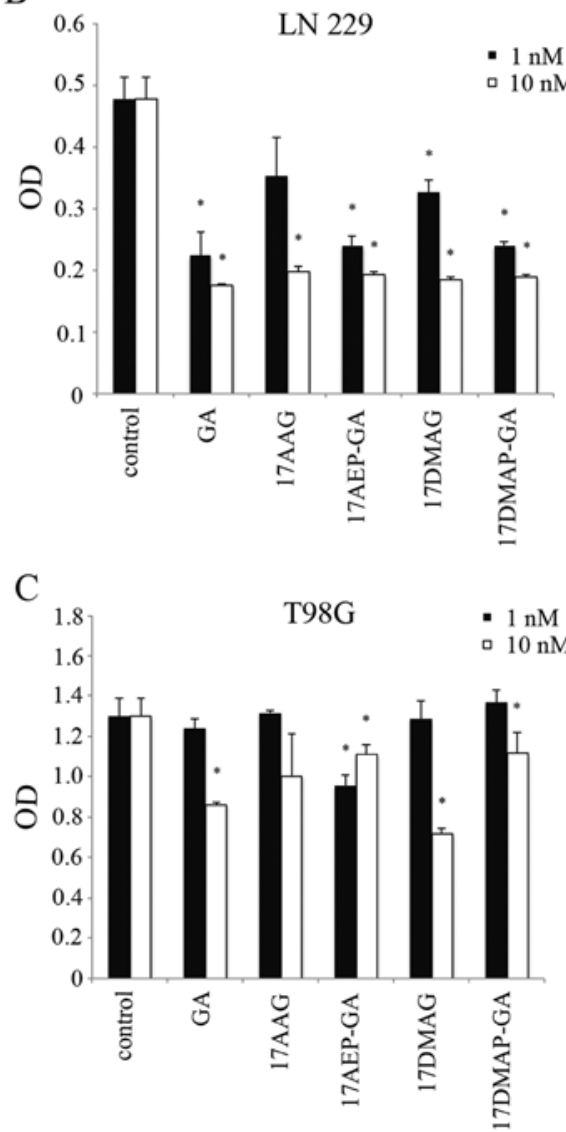

Figure 1. Proliferation of GBM cells after geldanamycin treatment. Cell proliferation was validated using CellTiter $96^{\circledR}$ AQueous One Solution assay. Cells were incubated for $96 \mathrm{~h}$ in different concentrations of GAs. (A) LN18, (B) LN229 and (C) T98G cell lines. Experiment was repeated twice in triplicates. $\mathrm{P}<0.05$ was considered statistically significant.

MET receptor. LN18 and T98G cell lines had a similar level of expression. There were no significant differences between tested cell lines. To determine whether MET receptor is functional on investigated cell lines we stimulated them by HGF and checked phosphorylation of MAPK 42/44 and AKT Ser 473 by western blot analysis (Fig. 3B). For all tested cell lines we observed strong phosphorylation of AKT and MAPK after 5 min of stimulation by HGF. However, LN18 cell line did not show any differences in AKT phosphorylation between control cells and cells after stimulation. It might suggest constitutive activation of AKT. 
A

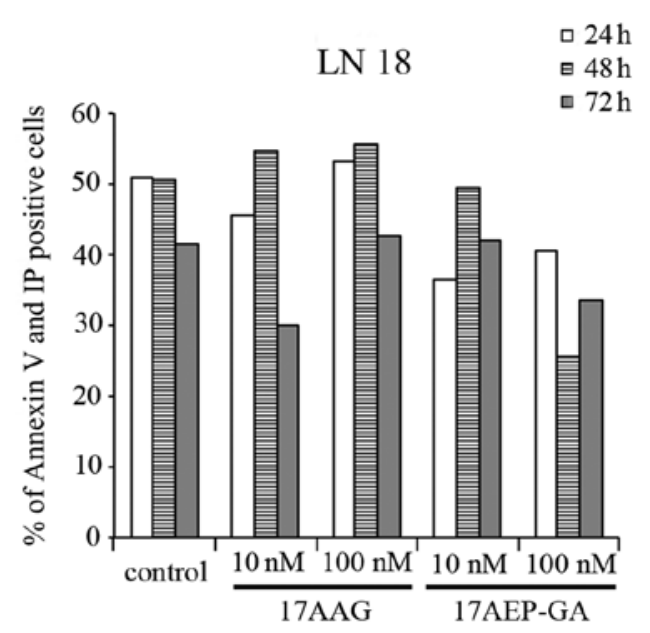

Annexin- $\mathrm{V}$ binding assay

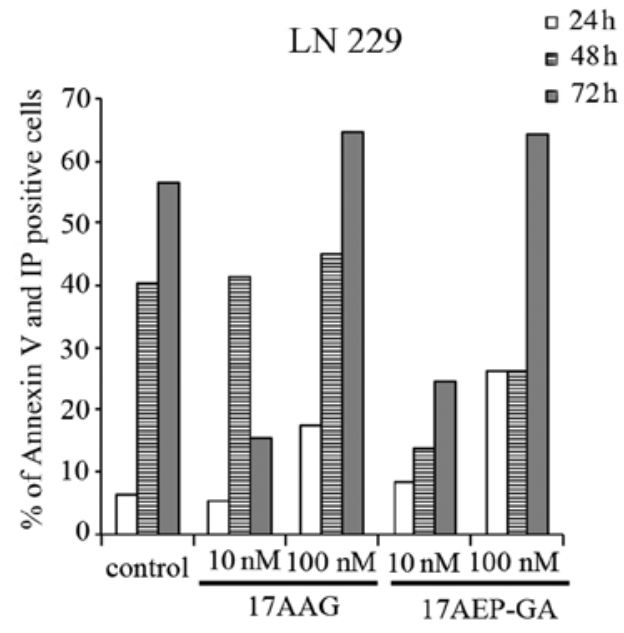

B

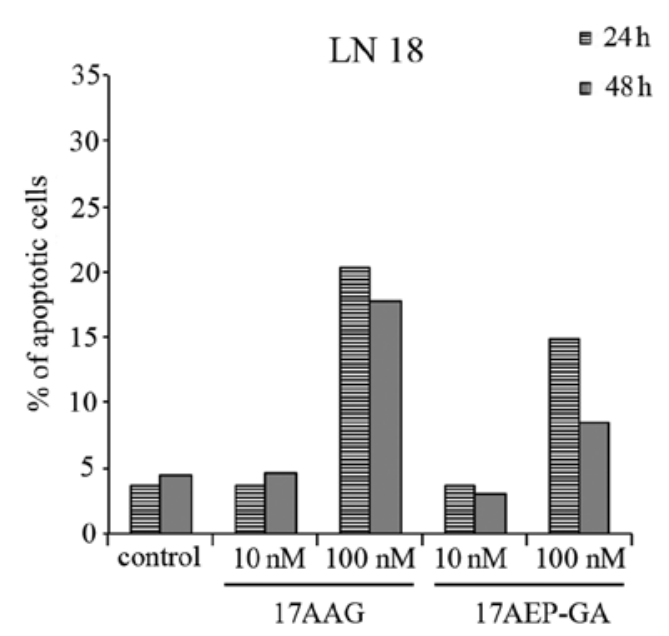

Caspase-3 activity

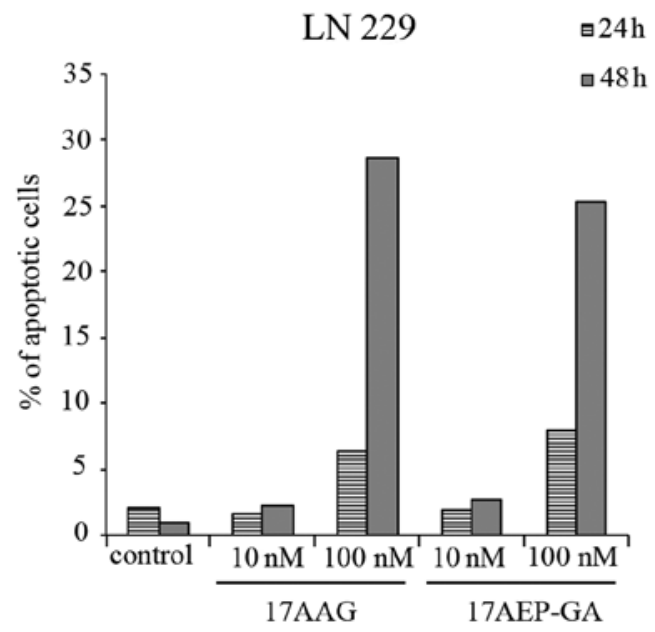

Figure 2. Geldanamycins induce apoptosis in GBM cell lines. Using cytofluorometric analysis the percentage of apoptotic cells was estimated. (A) Annexin V staining of GBM cell lines after 24-, 48- and 72-h treatment with different concentrations of 17-AAG and 17-AEP-GA. (B) Caspase-3 staining of GBM cell lines after 24- and 48-h treatment with different concentrations of 17-AAG and 17-AEP-GA. Experiments were repeated twice and representative staining is shown.

HSP90 inhibitors downregulate expression of MET receptor. It has been shown that MET receptor is a client protein for HSP90 $(14,19)$. Thus, we checked if incubation of GBM cells with GAs had any effect on the surface expression of MET receptor. Cells were grown in the presence of GAs for $24 \mathrm{~h}$ and the level of MET receptor expression was evaluated by cytofluorometric analysis. The downregulation of MET receptor expression was observed (Fig. 3C). The strongest reduction was in LN229 cell line. These results confirm that the expression of MET receptor is dependent on the presence of HSP90 protein.

Geldanamycin and its analogs inhibit HGF-dependent chemotaxis and invasion of glioblastoma cells. Since GBM expresses high level of MET receptor and its expression can be modulated by GAs, we tested the influence of GAs on chemotactic response of GBM cells towards HGF. The GBM cells were subjected to 10 and $100 \mathrm{nM}$ concentrations of GAs and their ability to migrate was evaluated. We observed decreased migration of treated GBM cells towards HGF gradient (Fig. 4A). This effect was further enhanced when cells were preincubated for $6 \mathrm{~h}$ with GAs (Fig. 4B).

We also evaluated invasive properties of GBM cells after GAs treatment. LN229 cell line was treated with inhibitors in the concentration of $100 \mathrm{nM}$. We observed a strong decrease in the ability of GBM to transmigrate through extracellular matrix proteins after HGF stimulation (Fig. 4C).

\section{Discussion}

Despite substantial research the GBM is still an untreatable disease. Thus, finding new ways of GBM treatment is necessary. In this study we tested GAs as new potential agents for GBM therapy. Our results showed that GAs were able to block proliferation of GBM cells and to induce apoptosis of tumor cells. Importantly, GAs were also able to inhibit migration and invasion of GBM cells toward HGF gradient. 
A

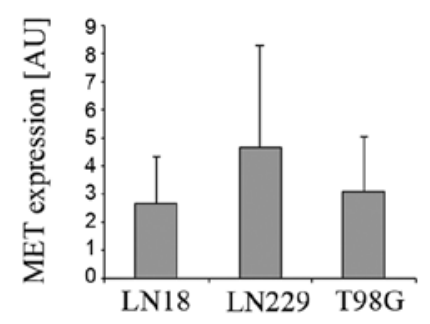

B

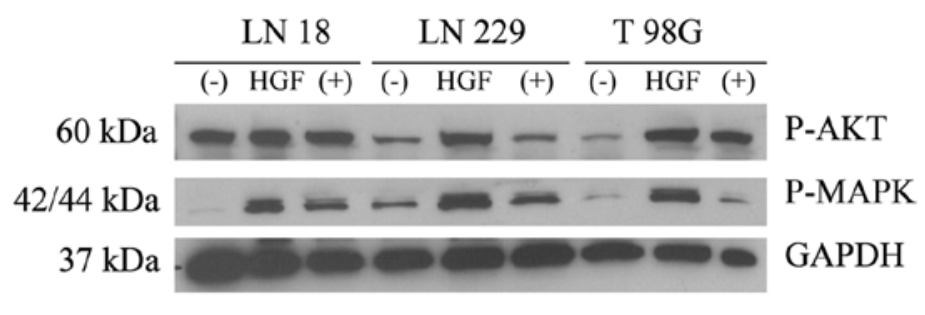

$\mathrm{C}$

control
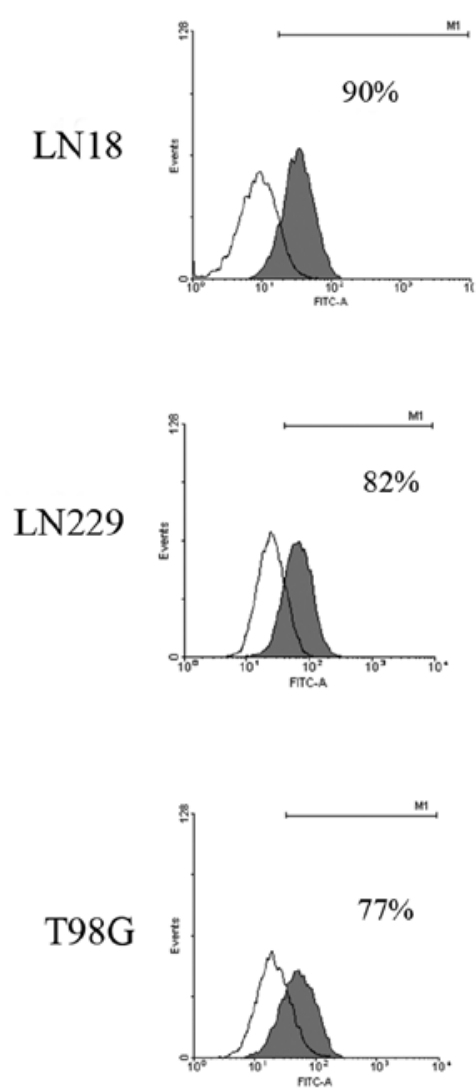
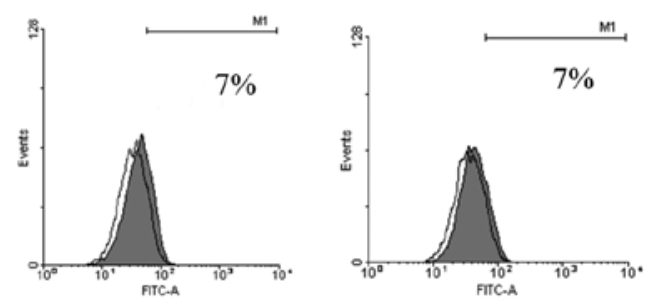

17AAG $100 \mathrm{nM}$
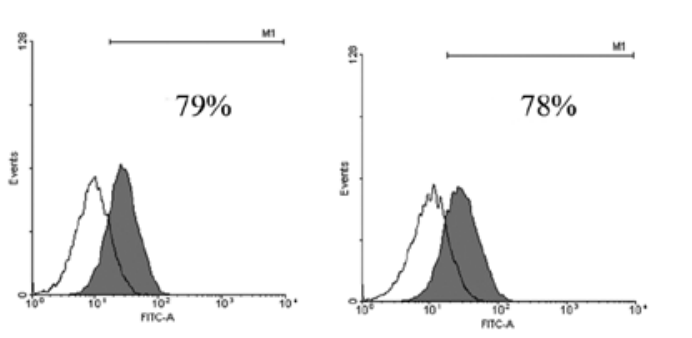

\section{AEP-GA $100 \mathrm{nM}$}
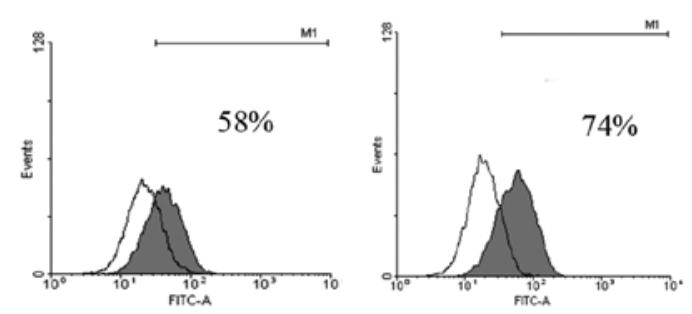

Figure 3. Expression and activation of MET receptor in GBM cell lines. (A) Real-time RT-PCR analysis of the level of mRNA MET receptor expression. (B) Western blot analysis of AKT and MAPK phosphorylation after HGF stimulation. GAPDH detection was used as a control of equal loading of the samples. A representative blot is shown. (C) Flow cytometry analysis of MET receptor expression after GAs treatment. Cells were incubated for $24 \mathrm{~h}$ in the presence of $100 \mathrm{nM}$ 17-AAG and 17-AEP-GA. All experiments were performed twice with similar results.

In this study we used five different HSP90 inhibitors, geldanamycin and its analogs. Some of these inhibitors e.g. 17-AAG is already in clinical trials (20) GA and 17AAG was also tested in GBM cells $(21,22)$.

All five inhibitors were able to inhibit proliferation and induced apoptosis of GBM cells. As we expected, based on our previous studies and published data (23), the inhibition of proliferation was observed already at the concentration of $1 \mathrm{nM}$ and achieving up to $50 \%$ for $10 \mathrm{nM}$, depending on a cell line (Fig. 1). The strongest inhibition was observed for LN18 and LN229 cell lines. However, contrary to other studies in which GAs at concentrations of $\geq 100 \mathrm{nM}$ were able almost completely to block growth of tumor cells $(14,23)$, we did not observe complete inhibition of GBM growth even at 1,000 nM concentration (data not shown). The efficiency of GA analogs in comparison to geldanamycin revealed the strongest inhibition of tumor cell proliferation in the case of 17-AEP-GA. This is in the agreement with our previous studies on rhabdomyosarcoma cell lines where we also observed the strongest inhibition of tumor cell proliferation with 17-AEP-GA (23).

The study of apoptosis revealed that the most effective HSP90 inhibitors that were able to induce apoptosis in GBM cells are 17-AAG and 17-AEP-GA. The concentration of $100 \mathrm{nM}$ induced apoptosis in LN18 and LN229 cell lines. Annexin $\mathrm{V}$ staining was confirmed by caspase-3 staining assay. These data show different effectiveness of tested analogs in inducing apoptosis in tumor cells $(14,23)$. In these experiments, 17-AEP-GA was again the most effective analog 

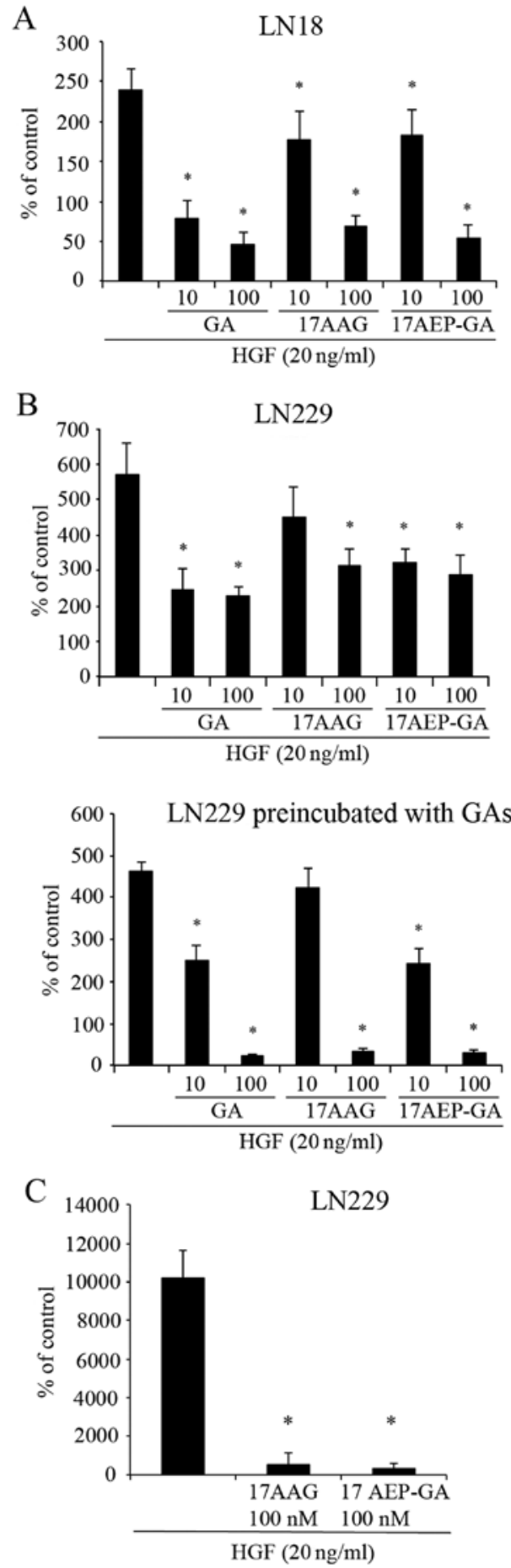

Figure 4. Migration and invasion of GBM cell lines after GAs treatment (A) Migration of LN18 cell line towards HGF $(20 \mathrm{ng} / \mathrm{ml})$ in the presence of 17-AAG and 17-AEP-GA (10 and $100 \mathrm{nM}$ ). (B) Migration of LN229 cell line towards HGF $(20 \mathrm{ng} / \mathrm{ml})$ in the presence of 17-AAG and 17-AEP-GA $(10$ and $100 \mathrm{nM}$ ) (left panel); migration of LN229 cell line towards HGF (20 ng/ $\mathrm{ml}$ ) after 6-h preincubation with 17-AAG and 17-AEP-GA (10 and $100 \mathrm{nM})$ (right panel). (C) Invasion of LN229 cell line towards HGF $(20 \mathrm{ng} / \mathrm{ml})$ in the presence of 17-AAG and 17-AEP-GA $(100 \mathrm{nM})$. Experiments were repeated twice in duplicates. (A and B) $\mathrm{P}<0.01 ;(\mathrm{C}) \mathrm{P}<0.001$.

among those tested. Based on our previous data and other publications we can postulate that the induction of apoptosis could be due to reduction in the expression of survival proteins including AKT that were shown to be the client proteins for HSP90, the direct target of GA and analogs $(13,23,24)$.
Interestingly, we also noticed some Annexin V-positive cells under control conditions. The reason for the presence of positive cells under control condition might be caused by a change in plasma membrane structure by surface exposure of phosphatidylserine (PS), while the membrane integrity remains unchallenged. Surface exposed PS can be detected by its affinity for Annexin V, a phospholipid binding protein (25).

MET receptor has been shown to be expressed on GBM cells and to induce various cellular responses in these cells (8). In this study, using real-time RT-PCR and flow cytometry, we showed that GBM cells express the MET receptor and that HGF stimulates the phosphorylation of MAPK and AKT kinases in GBM cells, two kinases involved not only in tumor growth but also in tumor cell migration $(26,27)$. Moreover, we have shown that GAs can downregulate the surface expression on MET receptor.

Next, we confirmed that HGF can stimulate the migration and invasion of GBM cells as shown previously $(8,28)$. In our previous studies on rhabdomyosarcoma cells we showed that HGF induced migration of these cells can be blocked by HSP90 inhibitors (19). Thus, in this study, we tested whether HSP90 inhibitors, such as GAs, can block the ability of GBM cells to migrate and invade. Strong inhibition of GBM migration and invasion was noted after treatment of tumor cells with 10 and $100 \mathrm{nM}$ of 17-AAG and 17-AEP that was comparable to inhibition observed with geldanamycin. This block of tumor cell migration is due to inhibition of MET receptor expression (mRNA expression and FACS analysis in this study). Similar results have been obtained for different tumors $(19,22,23,28)$. GAs are able to inhibit the expression of MAPK and AKT kinases which were shown previously to be involved in activating the motility of tumor cells $(26,27)$. Thus, parallel blocking of MET receptor expression and activation of kinases involved in cell migration by GAs could be responsible for observed inhibition of GBM migration and invasion after HGF stimulation.

GA and its analogs have been shown to block growth of tumor cells and the expression of various oncoproteins including tyrosine kinase receptors $(17,19,23)$. However, most of the studies on GBM cells were conducted using geldanamycin and its analog 17-AAG. Due to its high toxicity, geldanamycin cannot be considered a useful therapeutic $(23,29)$ and $17-A A G$ has also some drawbacks, e.g. it is insoluble in water. In this study, we tested other geldanamycin analogs with some characteristics preferable in clinical use such as water solubility. Particularly, 17-AEP-GA seems to possess the best clinical features. It is water-soluble and the efficacy of blocking tumor growth and migration is in similar range to geldanamycin and 17-AAG. Importantly, our previous studies showed that the use of 17-AEP-GA gives less toxic side effects against normal cells (23).

Based on our results presented here and our previous studies (23), we postulate that 17-AEP-GA could be considered as a new potential therapeutic for the treatment of glioblastoma multiforme.

\section{Acknowledgements}

This study was supported by research grant from the Polish Ministry of Science and Higher Education (NN 401 010036, N N 401 054839, N N401 142339). 


\section{References}

1. McLendon RE and Halperin EC: Is the long-term survival of patients with intracranial glioblastoma multiforme overstated? Cancer 98: 1745-1748, 2003.

2. Navas-Acién A, Pollán M, Gustavsson P, Floderus B, Plato N and Dosemeci M: Interactive effect of chemical substances and occupational electromagnetic field exposure on the risk of gliomas and meningiomas in Swedish men. Cancer Epidemiol Biomarkers Prev 11: 1678-1683, 2002.

3. Galloway TJ, Indelicato DJ, Amdur RJ, Swanson EL, Smith AA and Marcus RB: Second tumors in pediatric patients treated with radiotherapy to the central nervous system. Am J Clin Oncol 35: 279-283, 2011.

4. Krishnan S, Wade R and Moorman AV: Temporal changes in the incidence and pattern of central nervous system relapses in children with acute lymphoblastic leukaemia treated on four consecutive Medical Research Council trials, 1985-2001. Leukemia 24: 450-459, 2010.

5. Cordier S, Monfort C, Filippini G, et al: Parental exposure to polycyclic aromatic hydrocarbons and the risk of childhood brain tumors: The SEARCH International Childhood Brain Tumor Study. Am J Epidemiol 159: 1109-1116, 2004.

6. Molina JR, Hayashi Y, Stephens C and Georgescu MM: Invasive glioblastoma cells acquire stemness and increased Akt activation. Neoplasia 12: 453-463, 2010.

7. Sauvageot CM, Weatherbee JL, Kesari S, et al: Efficacy of the HSP90 inhibitor 17-AAG in human glioma cell lines and tumorigenic glioma stem cells. Neuro Oncol 11: 109-121, 2009.

8. Abounader R and Laterra J: Scatter factor/hepatocyte growth factor in brain tumor growth and angiogenesis. Neuro Oncol 7: 436-451, 2005.

9. Mazzone M and Comoglio PM: The Met pathway: master switch and drug target in cancer progression. FASEB J. 20: 1611-1621, 2006.

10. Lesko E and Majka M: The biological role of HGF-MET axis in tumor growth and development of metastasis. Front Biosci 13: 1271-1280, 2008.

11. Jiang W, Hiscox S, Matsumoto K and Nakamura T: Hepatocyte growth factor/scatter factor, its molecular, cellular and clinical implications in cancer. Crit Rev Oncol Hematol 29: 209-248, 1999.

12. Weidner KM, Sachs M and Birchmeier W: The Met receptor tyrosine kinase transduces motility, proliferation, and morphogenic signals of scatter factor/hepatocyte growth factor in epithelial cells. J Cell Biol 121: 145-154, 1993.

13. Pratt WB and Toft DO: Regulation of signaling protein function and trafficking by the hsp90/hsp70-based chaperone machinery. Exp Biol Med (Maywood) 228: 111-133, 2003.

14. Maulik G, Kijima T, Ma PC, et al: Modulation of the c-Met/ hepatocyte growth factor pathway in small cell lung cancer. Clin Cancer Res 8: 620-627, 2002.

15. Workman P: Altered states: selectively drugging the Hsp90 cancer chaperone. Trends Mol Med 10: 47-51, 2004.

16. Whitesell L, Mimnaugh EG, De Costa B, Myers CE and Neckers LM: Inhibition of heat shock protein HSP90-pp60v-src heteroprotein complex formation by benzoquinone ansamycins: essential role for stress proteins in oncogenic transformation. Proc Natl Acad Sci USA 91: 8324-8328, 1994.
17. Goetz MP, Toft DO, Ames MM and Erlichman C: The Hsp90 chaperone complex as a novel target for cancer therapy. Ann Oncol 14: 1169-1176, 2003.

18. Ochel HJ, Eichhorn K and Gademann G: Geldanamycin: the prototype of a class of antitumor drugs targeting the heat shock protein 90 family of molecular chaperones. Cell Stress Chaperones 6: 105-112,2001.

19. Lesko E, Gozdzik J, Kijowski J, Jenner B, Wiecha O and Majka M: HSP90 antagonist, geldanamycin, inhibits proliferation, induces apoptosis and blocks migration of rhabdomysoracoma cells in vitro and seeding into bone marrow in vivo. Anticancer Drugs 18: $1173-1181,2007$.

20. Ramanathan RK, Trump DL, Eiseman JL, et al: Phase I pharmacokinetic-pharmacodynamic study of 17-(allylamino)17-demethoxygeldanamycin (17AAG, NSC 330507), a novel inhibitor of heat shock protein 90 , in patients with refractory advanced cancers. Clin Cancer Res 11: 3385-3391, 2005.

21. Xie Q, Thompson R, Hardy K, et al: A highly invasive human glioblastoma pre-clinical model for testing therapeutics. J Transl Med 6: 77, 2008

22. Zagzag D, Nomura M, Friedlander DR, Blanco CY, Gagner JP, Nomura $\mathrm{N}$ and Newcomb EW: Geldanamycin inhibits migration of glioma cells in vitro: a potential role for hypoxia-inducible factor (HIF-1alpha) in glioma cell invasion. J Cell Physiol 196: 394-402, 2003.

23. Lukasiewicz E, Miekus K, Kijowski J, et al: High anti tumor activity against rhabdomyosarcoma cells and low normal cells cytotoxicity of heat shock protein 90 inhibitors, with special emphasis on 17- (2-(pyrrolidin-1-yl)ethyl)-aminno-17-demethoxygeldanamycin. J Physiol Pharmacol 60: 161-166, 2009.

24. Solit DB, Basso AD, Olshen AB, Scher HI and Rosen N: Inhibition of heat shock protein 90 function down-regulates Akt kinase and sensitizes tumors to Taxol. Cancer Res 63:2139-2144, 2003.

25. van Engeland M, Nieland LJ, Ramaekers FC, Schutte B and Reutelingsperger CP: Annexin V-affinity assay: a review on an apoptosis detection system based on phosphatidylserine exposure. Cytometry 31: 1-9, 1998

26. Hardy KM, Yatskievych TA, Konieczka J, Bobbs AS and Antin PB: FGF signalling through RAS/MAPK and PI3K pathways regulates cell movement and gene expression in the chicken primitive streak without affecting E-cadherin expression. BMC Dev Biol 11: 20, 2011.

27. Miekus K, Jarocha D, Trzyna E, Majka M: Role of I-TAC-binding receptors CXCR 3 and CXCR7 in proliferation, activation of intracellular signaling pathways and migration of various tumor cell lines. Folia Histochem Cytobiol. 48: 104-111, 2010.

28. Xie Q, Gao CF, Shinomiya N, et al: Geldanamycins exquisitely inhibit HGF/SF-mediated tumor cell invasion. Oncogene 24: 3697-3707, 2005 .

29. Supko JG, Hickman RL, Grever MR and Malspeis L: Preclinical pharmacologic evaluation of geldanamycin as an antitumor agent. Cancer Chemother Pharmacol 36: 305-315, 1995. 